\title{
Three-dimensional Ultrasound in Infertility
}

\author{
${ }^{1}$ Radu Vlãdãreanu, ${ }^{2}$ Mona Zvâncã \\ ${ }^{1}$ Professor of Obstetrics and Gynecology, ${ }^{2}$ Resident and Fellow in Obstetrics and Gynecology \\ Elias University Hospital, Obstetrics and Gynecology Department, 17, Marasti Street, 020322 \\ Bucharest, Romania \\ Correspondence: Radu Vlãdãreanu \\ Mailing address: Elias Emergency Hospital, Obstetrics and Gynecology Department, \\ 17, Mãrãoti Blvd. SE 1, 020322, Bucharest, Phone/fax: 3161640 \\ GSM: 0722351 081, Email: vladareanu@gmail.com
}

\begin{abstract}
Moving forward from the first approach of three dimensional ultrasound, as a new gadget for technicians, the method makes proof of its use in an increasing number of medical fields.

Aim: The present paper, and its graphic back-up, are intended as a visual testimony of the fact that $3 \mathrm{D}$ ultrasound is much more than a simple generator of "nice", commercial, images. Moreover, its use may be extended from obstetrics to gynecological pathology, especially in cases related to infertility.
\end{abstract}

Material and methods: we performed a review of the main local infertility causes and their perception by the three dimensional ultrasound, as compared to the conventional methods. General literature citations were completed with personal cases and images. In our research we used a Kretz - Voluson Expert 730 ultrasound machine with a full logistic equipment. Our database was completed over a period of 18 months and included various pathology, such as uterine anomalies, fibroids, polyps, ovarian cysts and many others.

Results: Three dimensional ultrasound represents the best tool in evaluating the uterine cavity, the endometrium, assessing its volume and vascularity pattern. It also offers a very good image of the uterine structure, the adnexal morphology and their relationship. It performs a thorough pelvic assessment by a single examination. Moreover, it may realize a histerosalpingography, which is as efficient as the radiological method, but easier and with less side effects.

Conclusions: Even though it is technically more difficult and time consuming, a good practice and a high quality ultrasound equipment offer a series of benefits over any other kind of investigation.

\section{INTRODUCTION}

Over the past decade there was a very important progress in digital technology, bringing along the development of the main diagnostic tool in most clinical fields, the ultrasound. With the increasing utility of the transvaginal ultrasound, the female pelvis became more and more accessible, a wider variety of gynecological pathology was open to diagnosis and treatment. Real time conventional scan and Doppler analysis are now part of the routine pelvic exam and an accurate evaluation allows gathering a number of anatomical and even histological details, in search for functional information, such as luteal phase deficiency or the implantation prognosis. In this field, the three dimensional ultrasound seemed a natural step. Initially regarded as an experimental investigation tool, with few clinical utilities, it soon developed into a complementary method for obstetrics, where the liquid environment surrounding the fetus makes it possible to achieve images that are mainly spectacular and commercial. On the other hand, three and four dimensional ultrasound (3D/4D) examination of the fetus offers a general surface image, with functional informations and even data about the internal organs, using the new technologies, such as STIC, minimum mode and reconstruction of the vascular structures. These technical acquisitions promoted 3D/4D ultrasound to a higher level, from a simple "gadget" for technicians, generator of "nice" images, to a useful diagnostic tool, with a very promising future.

Gynecological examination was initially doomed by the lack of a fluid environment. The first images obtained were at least disappointing. Only after the development of the new technologies and rendering modes it became possible to acquire valuable diagnostic images of the female genital organs. The most important advantage was the visualization of the coronal plane. It was a point of view completely new for pelvic examination, even though it is based on the well known fact that conventional ultrasound is a two-dimensional rendering mode of a three dimensional organ. Even with technical advances in gynecological 3D ultrasound, the coronal plane still remains the most important acquisition. ${ }^{1}$ The basis of the $3 \mathrm{D}$ examination in gynecology, derived straight from the surface rendering mode in obstetrics, consists in a progressive plane-by-plane twodimensional scan, with digital image reconstruction. Starting from this point there are several possible rendering modes:

\section{Thick-slice Rendering Mode}

It represents the basic acquisition type, generated by a single scan of a limited number of planes that create a "slice" in the target organ, centered by a region of interest. The thinner the slice, the better the quality of the image. On the other hand, selecting a limited territory may leave outside investigational 
area possible important diagnostic details. The key to a good, diagnostic image is a very good two-dimensional section. The main utility of this rendering mode in gynecology is the visualization of the endometrium. Better images are obtained with a thick, echogenic tissue, during the secretory phase. The acquisition should be in a perfect sagital plane and the result is a coronal plane histogram.

\section{Multiplanar Rendering Mode \\ (TUI - tomographic ultrasound imaging)}

This rendering mode is derived from tomography and consists in sectioning the volume into a pre-determined number of slices, which are successively displayed. The final images are conventional, but the acquisition is three-dimensional. The main advantage is that from a single scan we can obtain data regarding structures at different levels. Moreover, the examination time is consistently reduced. The number and thickness of the slices may be subsequently modified, without further patient examination. $^{2}$

\section{Inversion and Minimum Mode}

Inversion mode implies a photographic switch in the image aspect, such as transonic structures appear of solid consistency and the echogenic surrounding elements, appear as a liquid environment. The result is a better definition of the contours and relations with connecting structures in the region of interest. Moreover, by digital cutting, it is possible to alienate unwanted elements, rotate the section and create a very good anatomical image. ${ }^{3}$

Minimum mode enhances transonic structures, without changing their appearance in the final image. It shadows away solid organs, in order to visualize their fluid content.

This rendering mode is best employed for fluid structures such as ovarian cysts or follicles, as well as the uterine cavity during the saline infusion sonohysterography. The shape, the volume, as well as the print created by submucous myomas or polyps are easily detected.

\section{Volume Contrast Imaging ( $\mathrm{VCl})$}

Employed statically, as well as dynamically, this rendering mode is based on enhancing contrast and underlying the shape of solid structures. It can offer images in two planes:

- plane A (VCI-A), the conventional section plane, with limited use

- $\quad$ plane C (VCI-C), an image of the coronal plane. It offers a direct view of the coronal plane, with a contrast enhancement. ${ }^{4}$

\section{VOCAL II (virtual organ computer aided analysis)}

One of the newest ultrasound technologies, it was created for accurate volume calculations of solid or fluid structures. It starts with a basic static acquisition of the interest region, followed by a contour trace in two planes, while the volume is rotated in the third plane with a predetermined angle. The actual volume calculation is computer generated after the volume is completely traced. ${ }^{4}$ The acquisition may be improved by adding Doppler analysis, thus creating a vascular volume. There are a number of vascular parameters that can be digitally detected. This tool is extremely useful in generating an implantation prognosis in stimulated cycles, according to the type and degree of endometrial vascularity. ${ }^{5}$

\section{D and 4D Angiography}

It consists of a highly spectacular rendering mode of the vascular structures, either statically or dynamically. The acquisition is based on power Doppler or high density flow (HD flow). The diagnostic informations are strictly anatomical, descriptive, no functional data can be provided.

The image may be viewed as a simple angiography or integrated in the surrounding solid structure, as a "glass body" image, with very little details about the relations with the organ (Fig. 1).

There is a particular investigation in which 3D angiography is very useful, that is the saline infusion hysterosalpingography, when the fluid passage through the tubes may be perceived and rendered as a 3D Doppler image.

\section{MATERIAL AND METHOD}

In order to assess the benefit of 3D/4D ultrasound examination over different conventional methods, we used a Kretz - Voluson Expert ultrasound machine with full logistic equipment. Our selection of cases was completed over a period of 18 months, from October 2005, until March 2007. The purpose of this study was to achieve a wide variety of uterine and ovarian pathologies, documented with complementary diagnosis methods.

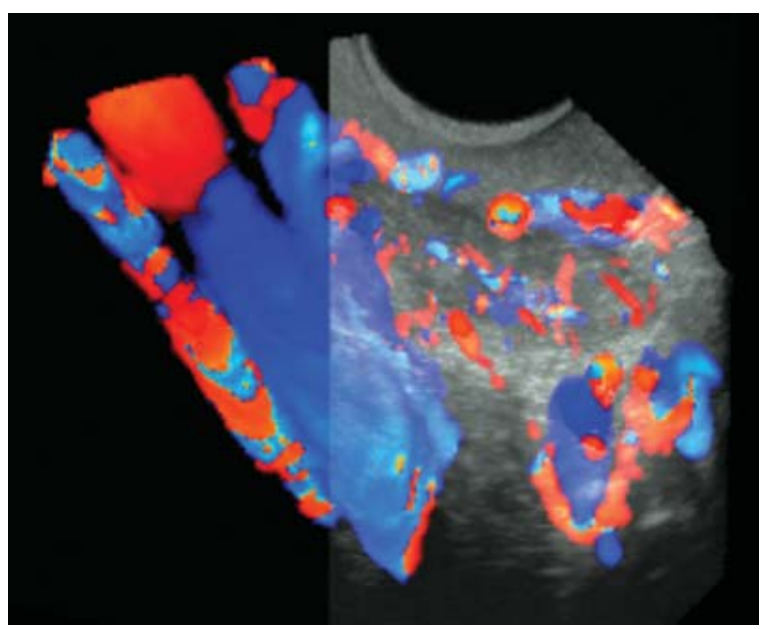

Fig 1: Three dimensional angiography of the iliac artery and the uterine artery origin. The image represents both 3D vascular rendering modes, angiography and "glass body" 
We focused mainly on young women with local causes of infertility, as it represents a more and more important category, where prompt and accurate diagnosis is essential.

We considered the standard definition of infertility as failure to conceive after 12 months of unprotected regular intercourse. Statistically, it affects almost $10 \%$ of the couples, with $40 \%$ of the cases related to female pathology. ${ }^{6}$ Male infertility is involved in a similar percentage and in $20 \%$ of the situations, the etiologies are intricate. Traditionally, infertility causes may be divided into cervical, uterine/endometrial, tubal and peritoneal infertility, and, finally, anovulation. All these territories will consist in targets for sonographic evaluation, conventional, as well as three dimensional. It is worth mentioning that over the past 20 years there has been a shift in the causes of infertility, passing from the ovarian and uterine anomalies, to tubal and male infertility factors. Mostly, this is due to the increasing frequency of tubal and pelvic surgery, ectopic pregnancies, use of intrauterine contraceptive devices, and, not to mention the more and more frequent pelvic inflammatory disease. Thus, there is a consequent increase in the number of couples which present with tubal infertility. Obstruction and damage of the fallopian tubes account for almost $35 \%$ of all infertility cases. ${ }^{7}$

The study included a total number of 124 patients, aged between 25 and 38 years. Without aiming for an accurate statistics, we selected for presentation only the most representative cases for different local pathologies associated with infertility. Most of our patients were secondary infertility ( 86 cases $-69.3 \%$ ), following miscarriages ( 42 cases $-48.8 \%$ ), terminations (16 cases $-18.6 \%$ ) or deliveries ( 28 cases $-32.5 \%$ ). Among them, we selected only those patients with uterine, tubal or ovarian anomalies, creating a study group of 92 women. Severe male infertility and chronic anovulation associated with endocrine anomalies represented exclusion criteria.

We performed a systematic review of the main local infertility causes, with comparison to similar data found in literature. Without aiming for an accurate statistics, we divided the cases according to the anomaly type and selected for presentation only the most representative cases for different local pathologies associated with infertility. The paper, along with its graphics, is meant as a visual testimony of the fact the 3D/4D sonography represents a useful tool for the diagnosis and prognosis of uterine and ovarian pathology associated with infertility.

\section{RESULTS}

\section{Uterine Pathology}

\section{Normal Uterus}

Normal uterus is easily assessed using 3D ultrasound, where the coronal plane gives a good image of the endometrial cavity, the surrounding myometrium and of the uterine external contour, a fact of most importance. Conventional ultrasound, with a thorough scan in both in sagital and transverse sections, offers an almost complete description of the uterus, endometrial thickness and vascularisation pattern. Three dimensional ultrasound does not substitute, but completes the examination by offering a complete image of the uterine cavity in one single acquisition, static rendering mode or VCI-C (Fig. 2), calculating the endometrial volume with VOCAL II, as well as vascularity indices (Fig. 3).

In some cases there is necessary to complement a simple sonography with saline infusion hysterography (SIS). A very handy technique, cheap, with minimum patient discomfort, and very important diagnostic data. The endometrial cavity is easily visualized, the contrast is greatly enhanced, it is helpful in differentiating between polyps and submucous myomas. It also facilitates the differential diagnosis between endometrial hyperplasia and carcinoma, along with the Doppler analysis. ${ }^{8}$ It may visualize and, up to some point, treat uterine adhesions. ${ }^{9}$ Techniquelly speaking, it is easy to perform, as a short outpatient procedure, does not require any kind of anesthesia, it is safe, avoids X-ray exposure, is well tolerated and performed
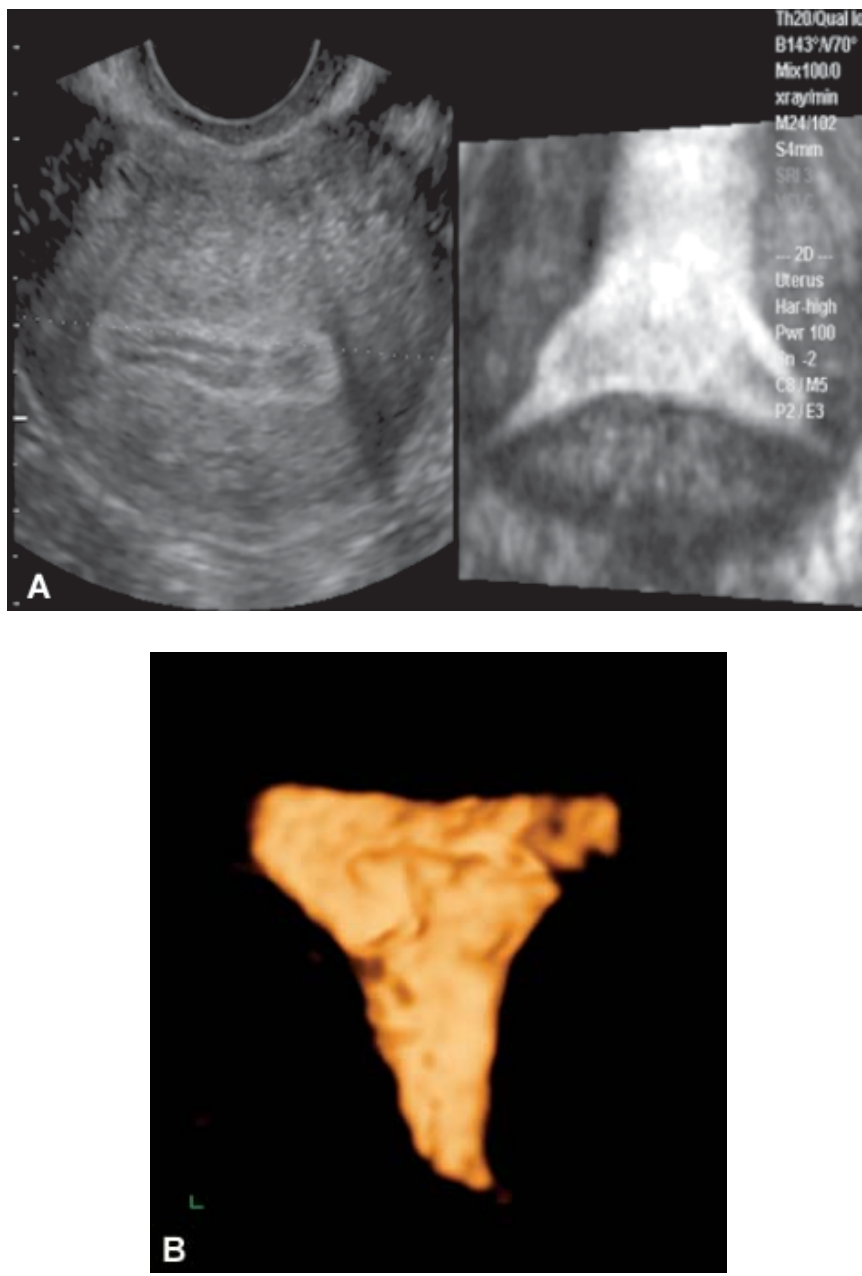

Figs $2 \mathrm{~A}$ and $\mathrm{B}$ : $\mathrm{VCl}-\mathrm{C}$ rendering mode; image of the endometrial cavity in a transverse acquisition (A). The endometrial cavity inversion mode during saline infusion. Offers the best data about the uterine cavity shape (B) 

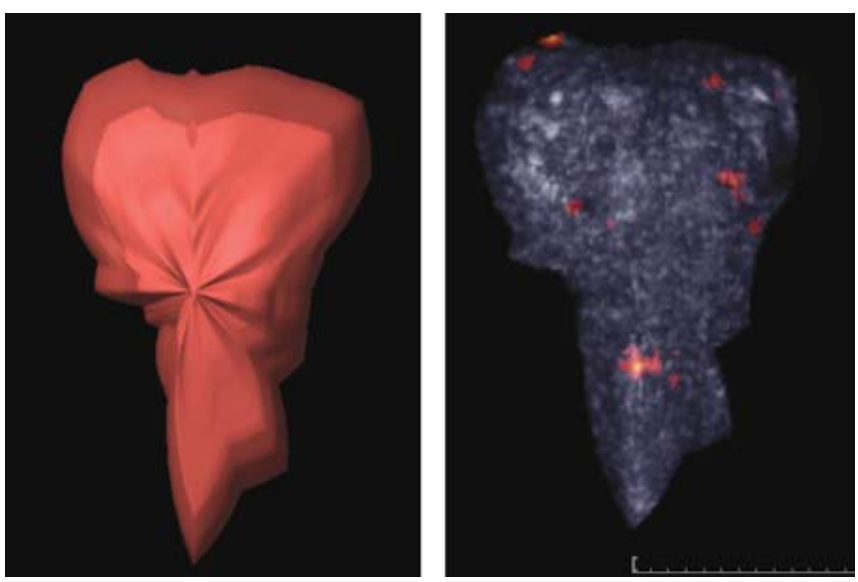

Fig. 3: VOCAL II - digitally reconstructed image of the endometrium, including a periendometrial region (shell), useful for vascularity indices calculations

at a minimal cost. ${ }^{10}$ The procedure is scheduled during the follicular phase, between days 5 and 10. It requires a sonohysterography catheter, such as the Goldstein Catheter (Cook ObGy), or a pediatric no.8 Foley catheter, which has the advantage of a very low cost, but is more difficult to position, as the balloon should be inflated at the internal os and not in the uterine cavity. Under transvaginal continuous evaluation, small amounts of saline solution are being injected in a pulsed manner. The sonographer gently slides the probe from one cornua to the other, then from the cervix to the fundus, reconstructing the uterine anatomy. 3D acquisition may be performed at any time, in a sagital or transverse section (Fig. 4). The image may be modified according to necessities, using inversion mode, rotation and digital cutting.

\section{The Endometrial Polyp}

Conventional ultrasound presents the endometrial polyp as a focal, unequal thickening of the endometrium, of higher echogenicity than the myometrium, with an easily detectable single feeding vessel. Large polyps may appear as diffuse endometrial thickening, being difficult to differentiate from simple hyperplasia. Three dimensional sonograpahy may facilitate diagnosis, using static acquisition, direct or inversion mode during SIS (Fig. 5). The differential diagnosis from a submucous myoma in questionable cases is easily set. Also, the shape, the dimensions, the origin and the impact on the endometrial cavity are clearly visualized, guiding the therapeutic procedure.

An alternative diagnostic method is hysteroscopy. With a $100 \%$ reliability it has the advantage of offering a therapeutic solution in one step. But it is an invasive procedure, it requires a very skilled practitioner, it may be associated with complications as uterine perforation, infection, hemorrhage and it may require general anesthesia.
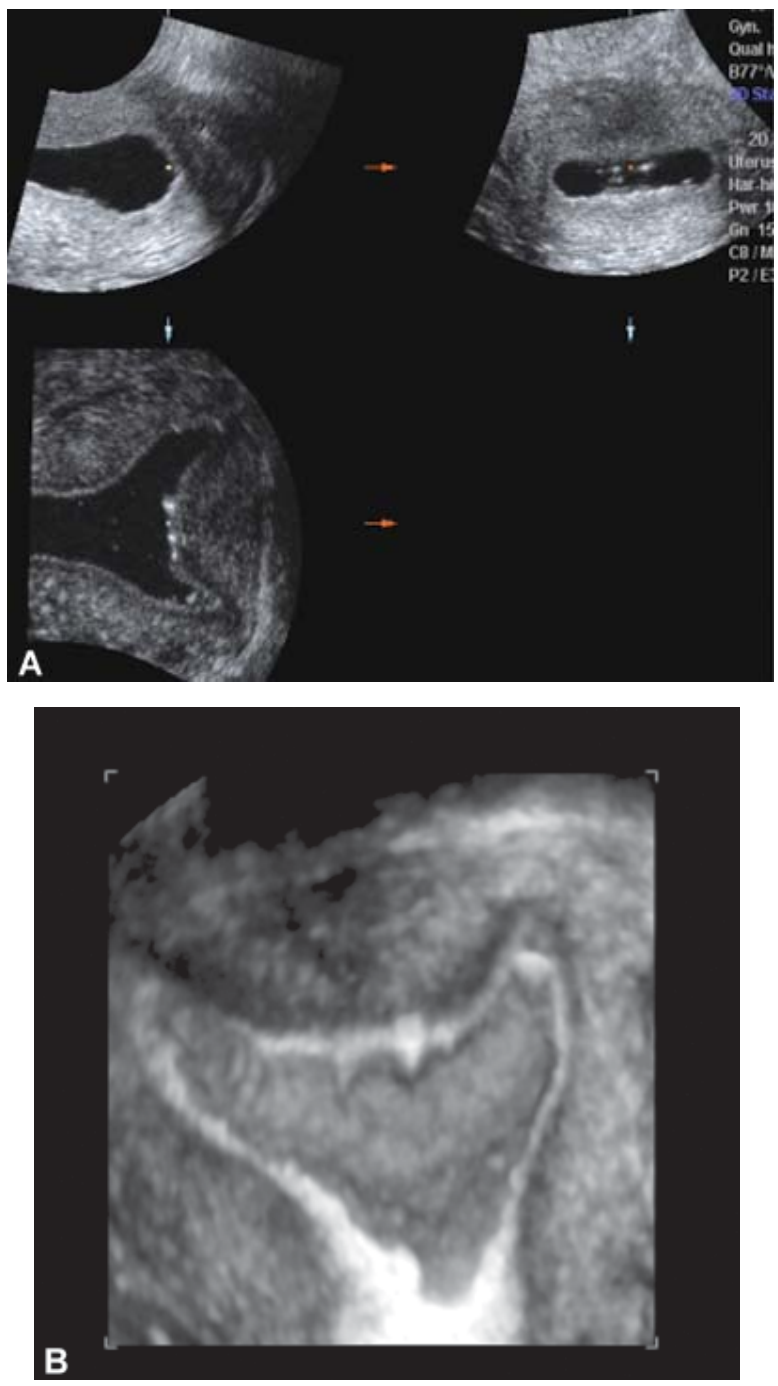

Figs $4 A$ and $B$ : Sectional planes of the uterus during saline infusion (A); the most important is the reconstructed coronal plane. The same patient, a different rendering mode $-\mathrm{VCl}-\mathrm{C}$ during saline infusion $(\mathrm{B})$

\section{Uterine Myoma}

Most frequent tumors of the feminine genital tract, uterine myomas may be generators of infertility by obstructing the fallopian tubes, distorting the endometrial cavity and causing subendometrial ischemia, thus interfering with gamete transport and implantation. ${ }^{11}$ Sonographically, they present as focal enlargements of the uterus, with a texture similar to the myometrium and posterior shadowing. The appearance greatly depends upon the presence of calcification or necrosis. Doppler ultrasound visualizes circular surface vessels, sometimes detecting the main feeding vessel. The resistance index is always relatively high, an average on $0.55 .^{12}$ The exact position, the impact on the ostium tubae and the uterine cavity may be difficult to assess by conventional ultrasound. A very easy 

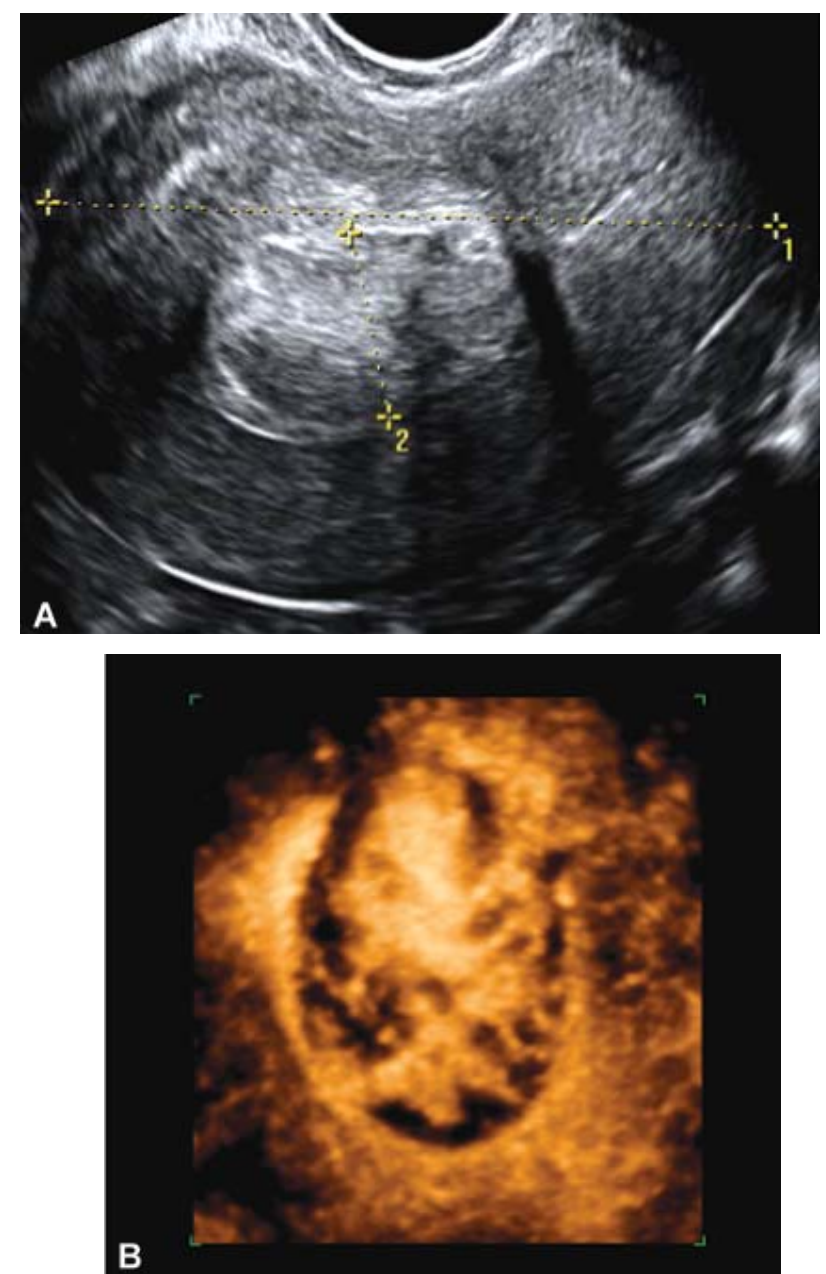

Figs $5 \mathrm{~A}$ and $\mathrm{B}$ : Large endometrial polyp - conventional transvaginal sagittal scan (A) and 3D image in the coronal plane (B)

solution in many cases is offered by a static 3D acquisition or static VCI-C. The increased echogenicity of the endometrium improves the visualization of the uterine cavity contour, but sometimes, especially in large myomas located on the anterior wall, this may be extremely difficult. Instillation of saline enhances contrast and delineates the uterine myoma. Inversion mode and rotation of the image may offer exact data regarding the dimension, position of the tumor and degree of distortion of the cavity. In the same procedure it is possible to assess tubal patency, by direct view of the fluid spillage through the fallopian tubes under Doppler control, or indirectly, by the easy depletion of the uterine cavity with fluid accumulation in the Douglas pouch.

3D with reconstruction of the volume creates a very good hysterographic image, without supplementary investigations. It guides the therapeutic procedure in cases deferred to hysteroscopic resection, by evaluating the degree of protrusion in the uterine cavity. In the same manner it, also, selects the cases to benefit from laparoscopic or classic myomectomy.

\section{Müllerian Anomalies}

The exact incidence of congenital uterine anomalies is difficult to determine since many women with such anomalies are not diagnosed, especially if they are asymptomatic, but it seems to be around 2 to $4 \%$ of live births. ${ }^{13}$ The prevalence is higher among women with adverse reproductive outcomes. As an example, the incidence of Müllerian anomalies among women with recurrent first trimester miscarriage or late first trimester second trimester miscarriage/preterm delivery was 5 to $10 \%$ and greater than $25 \%$, respectively, in another study. ${ }^{14}$ The most common type of Müllerian anomalies are uterine anomalies, with a distribution as follows: septate/arcuate uteri (90\%), bicornuate uterus (5\%), and didelphic uterus (5\%).

There is a special mention regarding the association with spontaneous first trimester abortions. Among all types of congenital uterine anomalies, the septate uterus presents the highest rate of miscarriage. ${ }^{15}$ Clinically, this is of greatest importance, as septate uterus is considered a "mild" anomaly and the differential diagnosis with bicornuate uterus, anomaly with a better fertility prognosis, is difficult.

An accurate diagnosis in all cases implies a very good visualization of the uterine cavity, with focus on the fundus, and a delineation of the uterine external contour. So far, the most commonly used diagnostic method was hysterosalpingography (HSG). It provides excellent view of the uterine cavity and cervical canal, informations related to tubal patency, but no data regarding the fundal shape. Moreover, it exposes the patient to ionizing radiation and requires an X-ray laboratory. Alternative method for external visualization of the uterus is laparoscopy. Expensive, invasive, gives no information regarding the endometrial cavity. MRI may be employed in certain cases, with very good results, but at high costs.

\section{Arcuate/Septate Uterus}

The septate/arcuate uterus develops from a defect in canalization or resorption of the midline septum between the two müllerian ducts. The degree of septation varies from a small midline septum to total failure in resorption resulting in a septate uterus with longitudinal vaginal septum. Partial and complete uterine septa are defined by the proximity of the septum to the internal os; the presence or absence of a complete or partial vaginal septum is not relevant to the classification. ${ }^{16}$ A septate or arcuate uterus has a normal external surface, but two endometrial cavities, in contrast to a bicornuate uterus which has an indented fundus and two endometrial cavities. The distinction between arcuate and septate uterus is rather difficult and up to some point, subjective. It is accepted that the arcuate uterus has a slight midline septum with a broad, fundal basis and normal external surface, while the septate uterus presents a more important septum and sometimes may have a small indentation that does not exceed $10 \mathrm{~mm}$ depth. 
Sonographically, the two uterine cavities are seen as split endometrial echoes, best visualized during secretory phase. The degree of septation may be assessed by conventional scan or, much better, by three dimensional sonography, thick slice or VCI-C. The coronal plane offers a very good diagnostic image of the endometrial cavity, as well as the fundal contour. In unclear cases, saline infusion gives a perfect image of the uterine cavity. 3D acquisition with inversion mode creates a hysterogram sometimes of better quality than the radiological image (Fig. 6).

In our study it was the most common Müllerian anomaly, with a total of 18 patients, confirmed by HSG or laparoscopy. All sonographic images were diagnostic, there were no false negative results. As a conclusion, apart from further pelvic evaluation, there is no need for more invasive testing.

\section{Bicornuate Uterus}

A bicornuate uterus refers to a uterus in which the fundus is indented (arbitrarily defined as $\geq 1 \mathrm{~cm}$ ) and the vagina is generally normal. ${ }^{17}$ This anomaly results from only partial fusion of the müllerian ducts. This leads to a variable degree of separation of the uterine horns that can be complete or partial. Characteristically, there is only one cervix. Thus, the diagnosis depends on the very good visualization of the two endometrial cavities and the cervix. From a theoretical point of view, it is easy to obtain a good coronal plane, but, sometimes, it may turn out to be quite difficult, due to some degree of uterine rotation. In our study group we had 3 patients with bicornuate uterus. We had a sonographic diagnosis in two cases. The third one was diagnosed as a septate uterus, rectified after the HSG. Saline infusion remains a very good method of clearing up a diagnosis, when necessary.

\section{Didelphic Uterus}

Uterine didelphys, or double uterus, occurs when the two müllerian ducts fail to fuse, thus producing duplication of the reproductive. Generally the duplication is limited to the uterus and cervix (uterine didelphys and bicollis [two cervices]) although duplication of the vulva, bladder, urethra, vagina, and anus may also occur. A complete vaginal septum, generating an obstructed hemivagina, may be associated with ipsilateral renal agenesis. ${ }^{18}$

Women with a didelphic uterus and bicollis often have good reproductive outcomes. A septated vagina occurs in $75 \%$ of cases and may cause difficulty with sexual intercourse or vaginal delivery. ${ }^{17}$

We encountered the anomaly in two of our patients, in one case associated with incipient pregnancy (Fig. 7). A correct diagnosis was stated in both cases using 3D ultrasound, with the remark that sonography cannot evaluate vaginal septum.

\section{The Ovaries}

Sonographic evaluation of the ovaries is directed towards their number, size, location, presence of dominant follicle; correlates
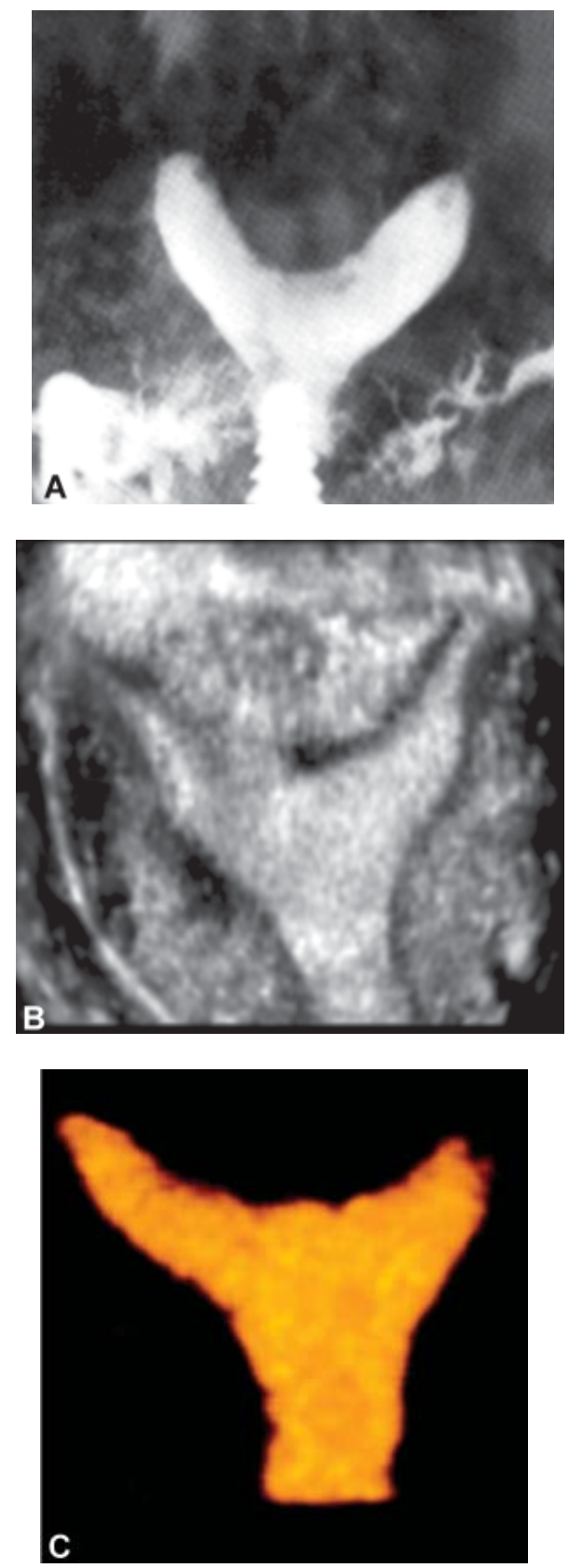

Figs $6 \mathbf{A}$ to $\mathbf{C}$ : Comparative images of the arcuate uterus: (A) radiological HSG; (B) VCl-C acquisition in the coronal plane; (C) inversion mode during SIS

follicle size to endometrial appearance and measurements; evaluates the cul-de-sac; assesses the corpus luteum, as a very important prediction factor for early pregnancy outcome; notes the presence of persistent follicular cysts. The ovarian volume is age dependent, as well as the follicular size and number and the degree of stromal vascularity. All these parameters may are easily assessed by 3D sonography, using the VOCAL II program. Their importance increased during the last decade, 

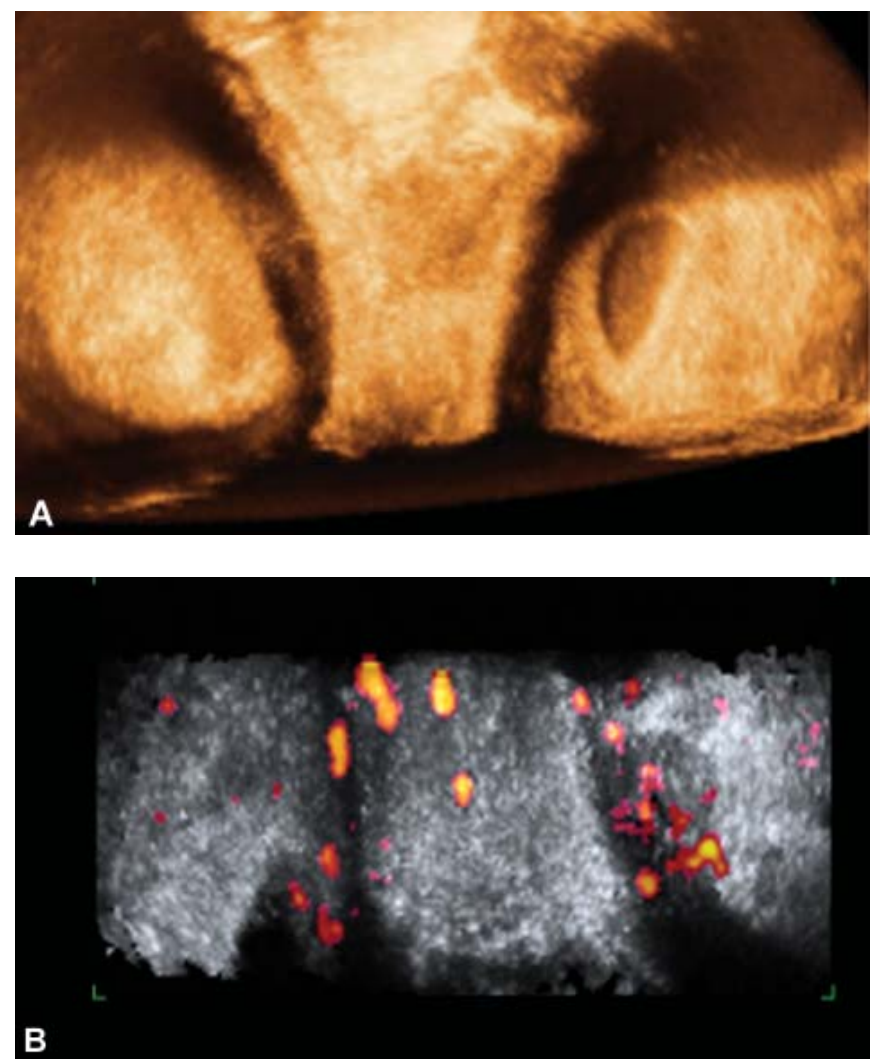

Figs 7A and B: Didelphic uterus with incipient pregnancy in one hemiuterus; comparative image of the static acquisition $(A)$ and "glass body" (B)

replacing the older hormonal parameter, such as the estradiol (E2) level, as predictor of follicular development and maturity. The follicular size may be expressed as a two-dimensional data, varying from 16-18 mm (gonadotropin stimulation) to 20-24 mm (clomiphene citrate stimulation), or as a volume of 3 to $7 \mathrm{ml}$. The stromal vascularization indices are calculated using the VOCAL II program after the follicular volume is taken out from the total ovarian volume. Resistance to ovarian artery blood flow begins to decline in the dominant ovary during the phase of rapid follicular growth, in association with rising serum estradiol concentrations, and reaches a nadir at the time of ovulation. Thereafter, it does not change for four to five days, and then gradually increases to a level slightly lower than that in the early follicular phase. This represents an important monitoring tool for stimulated cycles.

The most important ovarian event of luteal phase is represented by the development of the corpus luteum. Traditionally, a cystic structure of variable size, but less than $4 \mathrm{~cm}$, it can actually have many forms and the definitive element is the rich peripheral vascularization, described as a "fire ring". Sonographically, the corpus luteum is easily accessible. For a long time it was believed that progesterone levels were correlated with size and appearance of the corpus luteum, that is the proportion of the fluid component. Later it was proved that luteal function is related to the degree of thecal vascularization, characterized by a very low resistivity index (RI), which returns to normal 7 to 10 days after the ovulation in the absence of fecundation. Abnormal vascularization, with increased RI in the dominant ovary is a marker of luteal phase defect and a poor pregnancy outcome. ${ }^{20}$

The solitary ovarian cyst benefits of a correct volume calculation using 3D ultrasound and a graphic representation of the structure, its shape and relations. A cyst offers a fluid environment, facilitating the view of inner structures, such as intracystic papillae or vegetations, clearing the etiology and the prognosis. It interferes with fertility whenever a great proportion of ovarian substance is destroyed, but it represents one of the most frequent sonographic findings. The VOCAL II associated with Doppler acquisition offers informations about peripheral vascularization, as a supplementary prognosis index.

\section{The Fallopian Tubes}

Evaluating the tubal patency represents a key step in the assessment of the infertile couple, especially in situations with risk factors for tubal damage. Over the past 20 years there has been a shift in the causes of infertility, passing from the ovarian and uterine anomalies, to tubal and male infertility factors, obstruction and damage of the fallopian tubes accounting for almost $35 \%$ of all infertility cases. ${ }^{21}$ Normally, the fallopian tubes are not accessible to ultrasound evaluation, unless their diameter is increased by a pathological process, such as hydrosalpynx, pyosalpynx, ectopic pregnancy, tubal carcinoma or torsion. The diagnosis of tubal patency has changed very little during time, laparoscopy with chromo per tubation being still considered the "gold standard", as it was 20 years ago, along with radiological HSG. Even though it is not possible in all situations we consider that 3D ultrasound may represent a very good diagnostic tool, in cases with dilated tubes and a good image of the female pelvis. The most representative rendering mode is inversion mode, very spectacular and easy to handle, especially in patients with an amount of fluid in Douglas pouch.

A special remark has to be made regarding the evaluation of tubal patency in patients with non-dilated salpingae. Even though very little related to $3 \mathrm{D}$ ultrasound, saline infusion hysterosalpingography may beneficiate from its techniques. A variable amount of saline solution is injected in pulses, under continuous vaginal scan. Power Doppler permits a good image of the tubal passage (Fig. 8) and it may be combined with 3D acquisition, creating a graphic representation of the fallopian tube. In some cases during the saline passage the tube is dilated such as a static acquisition with inversion mode is possible, but this is rather accidental, as it requires a long saline pulse and an important tubal diameter.

With all its disadvantages, such as case-to-case variability, time consuming technique, high rate of false positive results, 


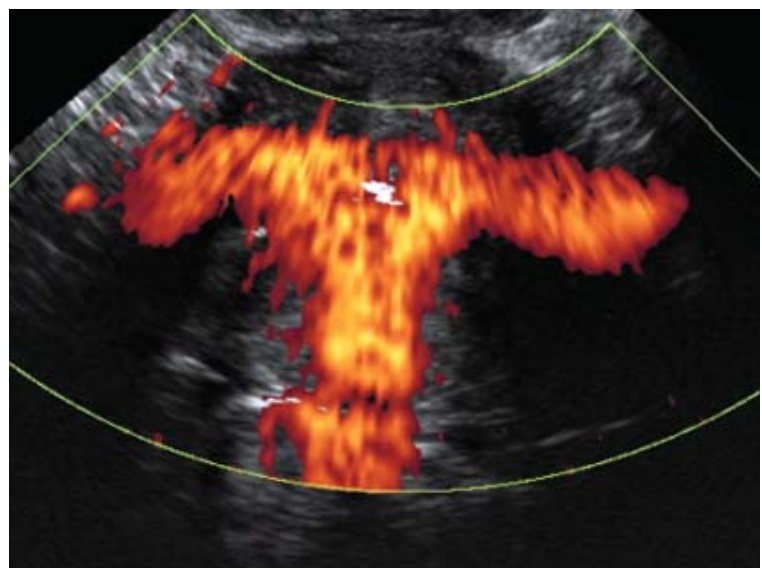

Fig. 8: Saline infusion hysterosalpingography under power Doppler control shows easy passage of the fluid through both fallopian tubes

the ultrasound evaluation of the fallopian tubes still represents the main developing direction for future tubal investigation.

\section{CONCLUSIONS}

Even though it seems the handiest diagnostic method, easily employed in out-patient clinics, an accurate investigation, especially in patients with minimal pathology, implies a lot of medical energy and time.

The first condition for a good image is a very performant ultrasound machine. Secondly, training and experience bring along more and more diagnostic details. It is difficult to talk about a learning curve, as acquisition and investigational techniques differ greatly from one case to another, one type of pathology to another. Definitely, a large experience with conventional ultrasound in female pelvis pathology is required, as well as a good acquaintance with 3D/4D technology. As in the obstetrics field, further technical development is expected to bring along easier and more comfortable ultrasound diagnostic methods. Until then, we can still conclude that sonography, in the hands of a skilled practitioner, with a good technological basis, is one of the best diagnostic tools in gynecology, successfully replacing more invasive methods.

\section{REFERENCES}

1. Donal B, Fenster A, Williams JC. Clinical utility of threedimensional Ultrasound. Radiographics 2000;20:559-71.

2. Benacerraf BR, Shipp TD, Bromley B. How Sonographic Tomography Will Change the Face of Obstetric Sonography: A Pilot Study. J Ultrasound Med 2005;24:371-8.

3. Baba K, Yo Y. Three dimensional ultrasound in Obstetrics and Gynecology. Tokyo, Japan; Medical View 2000.

4. Desser TS, Jeffrey RB. Tissue harmonic imaging techniques: physical principles and clinical applications. Semin Ultrasound CT MR 2001;22:31.
5. Mercé LT, Alcázar JL, Engels V, Troyano J, Bau S, Bajo JM. Endometrial volume and vascularity measurements by transvaginal three-dimensional ultrasonography and power Doppler angiography in stimulated and tumoral endometria: Intraobserver reproducibility. Gynecol Oncol 2006;100:544-50.

6. Kupesic S, Kurjak A, Bjelos D. Sonographic imaging in infertility. In: Donald School: Textbook of Ultrasound in Obstetrics and Gynecology. Eds: Kurjak A, Chervenak FA 2003, Parthenon Publishing Group.

7. Hill ML. Infertility and reproductive assistance. In: Neiberg DA, Hill LM, Bohm-Velez M, Mendelson EB (Eds). Transvaginal Ultrasound, St. Louis, Mosby Year Book 1992.

8. Soares SR, Barbosa dos Reis MM, Camargos AF. Diagnostic accuracy of sonohysterography, transvaginal sonography, and hysterosalpingography in patients with uterine cavity diseases. Fertil Steril 2000;73:406-10.

9. Salle B, Gaucherand P, de Saint Hilaire P, Rudigoz RC, Transvaginal sonohysterographic evaluation of intrauterine adhesions. J Clin Ultrasound 1999;27:131-5.

10. De Kroon CD, Jansen FW, Louwe LA, et al. Technology assessment of saline contrast hysterosonography. Am J Obstet Gynecol 2003;188:945-50.

11. Oliveira FG, Abdelmassih VG, Diamond MP, et al. Impact of subserosal and intramural uterine fibroids that do not distort the endometrial cavity on the outcome of in vitro fertilizationintracytoplasmic sperm injection. Fertil Steril 2004;81:582.

12. Kurjak A, Kupesic-Urek S, Miric D. The assessment of benign uterine tumor vascularization by transvaginal color Doppler. Ultrasound Med Biol 1992;18:645-9.

13. Grimbizis GF, Camus M, Tarlatzis BC, et al. Clinical implications of uterine malformations and hysteroscopic treatment results. Hum Reprod Update 2001;7:161-6.

14. Acien P. Incidence of Mullerian defects in fertile and infertile women. Hum Reprod 1997;12:1372-5.

15. Salim Daya. US evaluation of uterine anomalies. In: Jaffe, Person, Abramowicz (Eds). Imaging in infertility and reproductive endocrinology 1994.

16. Heinonen PK. Complete septate uterus with longitudinal vaginal septum. Fertil Steril 2006;85:700-3.

17. Troiano RN, McCarthy SM. Mullerian duct anomalies: imaging and clinical issues. Radiology 2004;233:19-23.

18. Stassart JP, Nagel TC, Prem KA, Phipps WR. Uterus didelphys, obstructed hemivagina, and ipsilateral renal agenesis: the University of Minnesota experience. Fertil Steril 1992;57:75661.

19. Kurjak A, Kupesic-Urek S, Schulman H, Zalud I. Transvaginal color flow Doppler in the assessment of ovarian and uterine blood flow in infertile women. Fertil Steril 1991;56:870-4.

20. Bourne, TH, Hagstrom HG, Hahlin, M, et al. Ultrasound studies of vascular and morphological changes in the human corpus luteum during the menstrual cycle. Fertil Steril 1996;65:753-9.

21. Hill ML. Infertility and reproductive assistance. In: Neiberg DA, Hill LM, Bohm-Velez M, Mendelson EB (Eds). Transvaginal Ultrasound, St. Louis, Mosby Year Book 1992. 


\section{Subscription information:}

\section{Annual subscription:}

Individual: Rs. 1600.00

$\$ 130.00$

$£ \quad 75.00$

Institutional: Rs. 3000.00

$\$ 160.00$

$£ \quad 100.00$

\author{
(national) \\ (international) \\ (international) \\ (national) \\ (international) \\ (international)
}

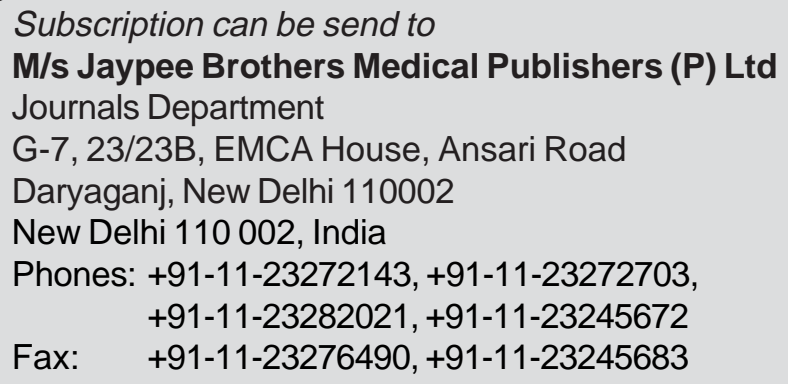

Subscription can be send to

M/s Jaypee Brothers Medical Publishers (P) Ltd Journals Department

G-7, 23/23B, EMCA House, Ansari Road

Daryaganj, New Delhi 110002

New Delhi 110 002, India

Phones: +91-11-23272143, +91-11-23272703,

$+91-11-23282021,+91-11-23245672$

Fax: $\quad+91-11-23276490,+91-11-23245683$

This journal is published quarterly, i.e. January-March, April-June, July-September and October-December, every year. Dollar rates apply to subscribers in all the countries except the UK and the Republic of Ireland where the pound/ sterling price applies. All subscriptions are payable in advance and all the rates include postage. Journals are sent by air to all the countries except Indian subcontinent. Subscriptions are on an annual basis, i.e. from January to December. Payment will be made by sterling cheque, dollar cheque, credit card or directly through our bank account at the following address:
1. Our banker's name
Canara Bank, Netaji Subhash Marg
Darya Ganj, New Delhi 110002
2. Telephone No:
011-23273015, 011-23273849
3. Fax No:
011-23255606
4. Telex Number
3166291
5. Our Current A/c. No.
3828
6. Amount to be transferred in the name of:
7. Swift code No.
JAYPEE BROTHERS MEDICAL PUBLISHERS (P) LTD., NEW DELHI
CNRB IN BB DFM

For further queries please do not hesitate to contact MR TARUN DUNEJA at the above address.

\section{JAYPEES JOURNAL ADVERTISEMENT RATES}

\section{(For the Print Issues)}

Page

Back cover-colour

Inside front cover-colour

Inside back cover-colour

Special position*-colour

Inside full page-colour

\begin{tabular}{lrl} 
& Single issue & \\
\hline Rs. 25,000 & $\$ 850.00$ & $€ 700$ \\
Rs. 20,000 & $\$ 750.00$ & $€ 500$ \\
Rs. 15,000 & $\$ 650.00$ & $€ 400$ \\
Rs. 12,500 & $\$ 600.00$ & $€ 350$ \\
Rs. 10,000 & $\$ 500.00$ & $€ 300$
\end{tabular}

*First page, page facing editorial board, page facing table of contents.

Cover page advertisements not available for a single issue.

\section{Technical Details}

Paper size

Print size

Digital file format

Printed on art paper using offset printing.

\section{Schedule}

Issues are published in the months of January, April, July and October.

Advertisement material along with purchase order and payment should reach us at least four weeks prior to the scheduled print date.

\section{Payment Details}

- Payment should favour "Jaypee Brothers Medical Publishers Pvt (L) " and should be payable at New Delhi, India

- Payment to be done at the time of submitting the advertisement material/booking the advertisement. Please send your advertisement request, payment and advertisement material to the address given above. Editorial board reserves the right to accept or decline the advertisement. 